\title{
Novel markers in pediatric-type follicular lymphoma
}

\section{Claudio Agostinelli ${ }^{1}$ - Ayse U Akarca ${ }^{2}$ - Alan Ramsay ${ }^{3} \cdot$ Hasan Rizvi $^{4} \cdot$ Manuel Rodriguez-Justo $^{2,3}$ - Sabine Pomplun ${ }^{3}$. Ian Proctor ${ }^{3}$ - Elena Sabattini ${ }^{1}$ - David Linch $^{5}$ - Stephen Daw ${ }^{6}$. Stefania Pittaluga ${ }^{7}$ - Stefano A Pileri ${ }^{8}$ - Elaine S Jaffe ${ }^{7}$. Leticia Quintanilla-Martinez ${ }^{9} \cdot$ Teresa Marafioti $^{2,3}$}

Received: 24 March 2019 / Revised: 29 April 2019 / Accepted: 31 May 2019/Published online: 4 November 2019

(C) The Author(s) 2019

\begin{abstract}
The aim of this study was to review the histopathological, phenotypic, and molecular characteristics of pediatric-type follicular lymphoma (PTFL) and to assess the diagnostic value of novel immunohistochemical markers in distinguishing PTFL from follicular hyperplasia (FH). A total of 13 nodal PTFLs were investigated using immunohistochemistry, fluorescence in situ hybridization (FISH), and PCR and were compared with a further 20 reactive lymph nodes showing FH. Morphologically, PTFL cases exhibited a follicular growth pattern with irregular lymphoid follicles in which the germinal centers were composed of numerous blastoid cells showing a starry-sky appearance. Immunohistochemistry highlighted preserved CD10 (13/13) and BCL6 (13/13) staining, CD20 (13/13) positivity, a K light chain predominance (7/13), and partial BCL2 expression in 6/13 cases (using antibodies 124, E17, and SP66). The germinal center (GC)-associated markers stathmin and LLT-1 were positive in most of the cases (12/13 and 12/13, respectively). Interestingly, FOXP-1 was uniformly positive in PTFL (12/13 cases) in contrast to reactive GCs in FH, where only a few isolated positive cells were observed. FISH revealed no evidence of $B C L 2, B C L 6$, or MYC rearrangements in the examined cases. By PCR, clonal immunoglobulin gene rearrangements were detected in $100 \%$ of the tested PTFL cases. Our study confirmed the unique morphological and immunophenotypic features of PTFL and suggests that FOXP-1 can represent a novel useful diagnostic marker in the differential diagnosis between PTFL and FH.
\end{abstract}

Keywords Pediatric-type follicular lymphoma $\cdot$ Immunohistochemistry $\cdot$ Differential diagnosis $\cdot$ FOXP-1

\section{Introduction}

Pediatric-type follicular lymphoma (PTFL) is a distinct clinicopathological entity in the revised 4th edition of the WHO Classification of Tumours of Haematopoietic and Lymphoid Tissues [1].

Claudio Agostinelli and Ayse U Akarca contributed equally to this work.

Teresa Marafioti

t.marafioti@ucl.ac.uk

1 Haematopathology Unit, Department of Experimental Diagnostic and Specialty Medicine, S. Orsola-Malpighi Hospital, University of Bologna, Bologna Italy

2 Department of Pathology, University College London, London UK

3 Department of Cellular Pathology, University College Hospital London, London UK

4 Department of Cellular Pathology, Barts Health NHS Trust, London UK
Although PTFL was initially identified in pediatric patients, accounting for approximately $1-2 \%$ of all pediatric non-Hodgkin lymphomas (NHL), it was subsequently found to occur also in young adults and more rarely in older patients [1-12]. The median age at onset ranges from 7.5 to 14 years, and the disease shows a male predominance (male to female

5 Department of Haematology, University College London Cancer Institute, London UK

6 Children and Young People's Cancer Service, University College Hospital London, London UK

7 Haematology section, Laboratory of Pathology, Center for Cancer Research National Cancer Institute, Bethesda MD USA

8 Division of Haematopathology, European Institute of Oncology, University Hospital of Tübingen, Institute of Pathology, Tübingen Germany

9 University Hospital of Tübingen, Institute of Pathology, Tübingen Germany 
ratio $>10: 1)$ [1-12]. PTLF is currently regarded as a nodal disease that most commonly arises in head and neck lymph nodes and less frequently involves inguinal and axillary nodes $[1,10,11]$. Because of differences in histopathologic features, molecular profile, and clinical behavior, the revised 4th edition of WHO classification excluded from this category extranodal follicular lymphoma (FL) cases involving testis, epididymis, gastrointestinal tract, and kidney, which had been previously reported as PTFL [1]. Another entity that should be excluded is the new provisional entity "large B-cell lymphoma (LBCL) with IRF4 rearrangement," [1, 13-15] which usually presents in Waldeyer's ring and/or cervical lymph nodes but can also arise in the gastrointestinal tract; this lesion may be exclusively follicular, follicular and diffuse, or diffuse.

Most PTFL patients present with localized disease and after local excision show complete remission with excellent prognosis and disease-free survival; for this reason, a "watch and wait" strategy is currently recommended [1-9]. Histologically, the neoplastic follicles in PTFL are large and irregularly expanded, sometimes coalescent and are highly proliferative with prominent tingible body macrophages. Although largely meeting the current histological criteria for conventional grade 3B FL, a proportion of cases lack classical centroblasts and centrocytes and consist instead of medium-sized blastoid cells [1]. The revised 4th $\mathrm{WHO}$ classification indicates that $B C L 2$ rearrangements are not present in PTFL [1]; however, BCL2 protein expression has been reported in a minority of cases, usually with weak intensity [1]. PTFL also lacks $B C L 6, I R F 4$, and $M Y C$ rearrangements $[1,10,11]$. The molecular profile of PTFL differs from that of conventional $\mathrm{t}(14 ; 18)^{+}$and $\mathrm{t}(14 ; 18)^{-} \mathrm{FL}$, as PTFL is characterized by a low genomic complexity and lacks or has only rare mutations in the histonemodifying genes CREBBP, EZH2, and KMT2D, commonly found in conventional FL [10-12]. Furthermore, TNFRSF14 and MAP2K1 mutations are the most frequently reported genetic aberrations in PTFL [10-12]. A hot spot mutation in IRF8 (K66R, p.L66A), although less frequently reported, seems unique in PTFL [16]. In spite of the present knowledge of this condition, some cases present a challenge in the differential diagnosis with florid follicular hyperplasia $(\mathrm{FH})$, pediatric nodal marginal zone lymphoma (NMZL), and other $\mathrm{t}(14 ; 18)^{-}$FLs. Our purpose was to undertake a histopathological review and perform phenotypic and molecular analyses of a series of PTFLs, to assess the potential diagnostic value of novel markers which could assist in differentiating PTFL from FH.

\section{Material and methods}

\section{Tissue samples}

Formalin-fixed, paraffin-embedded (FFPE) tissue blocks of 37 cases originally diagnosed as PTFL were retrieved from the files of the Department of Histopathology, University College Hospital, London (UK); Department of Pathology, Birmingham (UK); and the Unit of Haematopathology, S. Orsola-Malpighi Hospital, University of Bologna (Italy). In addition, 20 cases of reactive lymph nodes with FH of children and young adults diagnosed at the Department of Histopathology, University College Hospital London, were included in the study as controls.

The 37 PTFL cases were reviewed by expert hematopathologists (TM, LQF, SAP, ESJ). A consensus diagnosis of PTFL was reached in 13 out of the 37 cases, by strict adherence to the following criteria of the revised WHO classification: (a) nodal disease, (b) pure follicular growth pattern with lack of diffuse areas, (c) morphology characterized by large expansile highly proliferative follicles often consisting of blastoid germinal center cells rather than classic centroblasts or centrocytes, (d) BCL6 expression with associated BCL2 negativity or weak positivity and high proliferative fraction $(>30 \%)$ by immunohistochemistry, (e) absence of $B C L 2, B C L 6$, and $M Y C$ rearrangements as well as $B C L 2$ amplifications [1]. To further confirm the neoplastic nature of the process, at least one of the following parameters was required: detection of IGH and/or IGK gene rearrangements. Cases characterized by $\mathrm{IRF}^{+}$follicles, in the absence of a negative FISH analysis of the corresponding gene, were excluded from the study to avoid possible inclusion of cases of LBCL with IRF4 rearrangement.

\section{Antibodies and immunohistochemistry}

Antibodies raised against fixation resistant epitopes were used for the detection of CD20 (mouse, clone L26, Dako, Ely, UK), CD3 (mouse, clone LN10, Leica Microsystems, Newcastleupon-Tyne, UK), CD10 (mouse, clone 56C6, Leica Microsystems, Newcastle-upon-Tyne, UK), BCL6 (mouse, clone GI191E/A8, CNIO, Madrid), BCL2 (mouse, clone 124, Dako, Ely, UK), BCL2 (Rabbit, clone E17, Menarini Diagnostics, Wokingham, UK), BCL2 (rabbit, clone SP66, Spring Bioscience, Pleasanton, CA, USA), IRF4/MUM1 (mouse, clone MUM1p, kindly provided by Prof. Brunangelo Falini, Perugia, Italy), IRTA-1 (mouse monoclonal, kindly provided by Prof. Brunangelo Falini, Perugia, Italy), c-MYC (rabbit, clone Y69, Epitomics), IgM (mouse polyclonal, Dako A/S, Glostrup, Denmark), IgD (mouse polyclonal, Dako A/S, Glostrup, Denmark), kappa (mouse polyclonal, Dako A/S, Glostrup, Denmark) and lambda (mouse polyclonal, Dako A/S, Glostrup, Denmark) light chains, CD21 (mouse, clone 1F8, Dako A/S, Glostrup, Denmark), forkhead box protein P1 (FOXP-1) (mouse, clone JC12 AbD Serotec, Oxford, UK), stathmin (STMN1) (rabbit, clone SP49, Spring Bioscience, Pleasanton, CA, USA), lectin-like transcript 1 (LLT1) (goat polyclonal, R\&D Systems), and Ki67/MIB1 (mouse, clone MIB1, Dako, Ely, UK). Immunostaining was performed using a BOND-III 
AutoStainer (Leica Microsystems, Newcastle-upon-Tyne, UK) as previously described [17]. A cutoff of staining > $30 \%$ of the examined cells was assigned as positive score, according to formerly defined criteria [18].

\section{FISH}

Interphase fluorescent in situ hybridization (FISH) for the detection of BCL2, BCL6, IRF4, and MYC chromosomal alterations was performed using Vysis (Abbott Italia, Rome, Italy) LSI $B C L 2$ dual color, break apart rearrangement probe (18q21); Vysis LSI BCL6 (ABR) dual color, break apart rearrangement probe (3p27); Kreatech (Leica Microsystems, Buccinasco, Milano, Italy) IRF4 dual color, break apart rearrangement probe (6p25.3); and Vysis LSI MYC dual color, break apart rearrangement probe (8q24) (all Abbott, Abbott Park, IL, USA). The cutoff value for the diagnosis of each probe set was the mean percentage of cells with a falsepositive signal constellation plus 3 standard deviations, as assessed on tissue from reactive lymph nodes. The break apart/split signal rearrangement probes also detect loss or amplification of the respective genes. FISH procedure was performed according to published standard methods $[19,20]$.

\section{DNA extraction and clonality analysis}

Sections of 5- $\mu \mathrm{m}$ thick were prepared from paraffin-embedded biopsy specimens. Samples were first deparaffinized in histoclear and then rehydrated in ethanol. Tissue digestion was carried out in $200 \mu \mathrm{l}$ of a solution containing $20 \mu \mathrm{l}$ of proteinase $\mathrm{K}$ in $180 \mu \mathrm{l}$ ATL Buffer (Qiagen); subsequently, the material was incubated at $56{ }^{\circ} \mathrm{C}$ overnight. Finally, DNA purification was performed by using a Qiagen DNA mini kit according to the manufacturer's instructions. DNA sample concentration and quality were assessed by spectrophotometry (260/280 nm using the NanoDrop). Only cases with a 260/280 nm ratio between 1.8 and 2 and 260/230 ratio of about 2.2 were considered evaluable. IGH and IGK gene rearrangements were analyzed by multiplex polymerase chain reaction (PCR) amplification according to the BIOMED-2 multiplex PCR protocol as previously reported [21].

\section{Results}

\section{Patients}

According to the criteria of the revised WHO classification [1], 13 out the original 37 cases were included in the study as PTFLs along with 20 FHs. After clinical, pathological, and immunophenotypic review, the 24 excluded cases comprised 6 inadequate samples, 8 cases re-classified as pediatric-type marginal zone lymphoma, 5 cases recognized as conventional FL (these cases showed BCL2 rearrangement), and 2 cases with extranodal localization involving the testis and conjunctiva. Two cases, which the histology and phenotype suggested PTFL, were excluded since neither rearrangement of the IGH and/or IGK genes was detected. An additional case was excluded after immunohistochemical review due to a diffuse and strong expression of IRF4, along with CD10 and BCL6, in the absence of IRF4 FISH analysis.

All patients included in the study were male, with an age range between 6 and 24 years and a median age of 15 years (Table 1). All cases were nodal in presentation: 6 in the head and neck region, 4 in the inguinal region, and 1 in the axilla (Table 1). For the remaining 2 cases, no specific anatomic site was recorded.

\section{Histological and immunophenotypic findings}

The 13 selected PTFLs showed a purely follicular growth pattern with irregular, often closely packed lymphoid follicles, many of which exhibited serpiginous outlines and attenuated mantle zones (Fig. 1a). The GCs lacked normal polarization while retaining a starry-sky appearance, and consisted of a monotonous proliferation of medium- to large-sized blastoid cells with round/oval nuclei, finely clumped chromatin, and small nucleoli (Fig. 1a inset) as distinct from typical centrocytes and centroblasts. Where intact lymph nodes were sampled, it was not uncommon to see small reactive follicles at the periphery of the specimen; the appearances of these two distinct areas, reactive follicles and enlarged abnormal follicles, result in the so called "node within a node" pattern (Fig. 1b). Extra capsular extension of neoplastic follicles was not observed. None of the cases showed areas with a diffuse growth pattern, although focal confluent follicles were noted. In some cases, a narrow peripheral rim of paler cells resembling "monocytoid differentiation" was seen around the expanded follicles; however, areas of true monocytoid B cell hyperplasia were lacking.

On immunophenotyping, the neoplastic follicles in PTFL were strongly and homogeneously $\mathrm{CD} 20^{+}(13 / 13), \mathrm{CD} 10^{+}$ $(13 / 13)$, and BCL6 ${ }^{+}(13 / 13)$, with very few or no atypical cells in the interfollicular areas (Table 1) (Fig. 1c-e). BCL2 protein expression was seen in 6/13 (46\%) cases, although the staining was weak and heterogeneous, varying in intensity between different follicles (Fig. 1f). None of the PTFL cases demonstrated expression of the MYC protein (Table 1). Ki-67 showed a high proliferation fraction often with accentuation at the periphery of the GCs. IgD and CD21 highlighted the attenuated mantle zones and expanded irregular, though not fragmented, follicular dendritic cell (FDC) meshworks respectively (Fig. 1g). Staining for immunoglobulin heavy and light chains was carried out in most cases (in few instances, no additional sections could be cut due to tissue exhaustion). The results showed expression of intracytoplasmic IgM in 


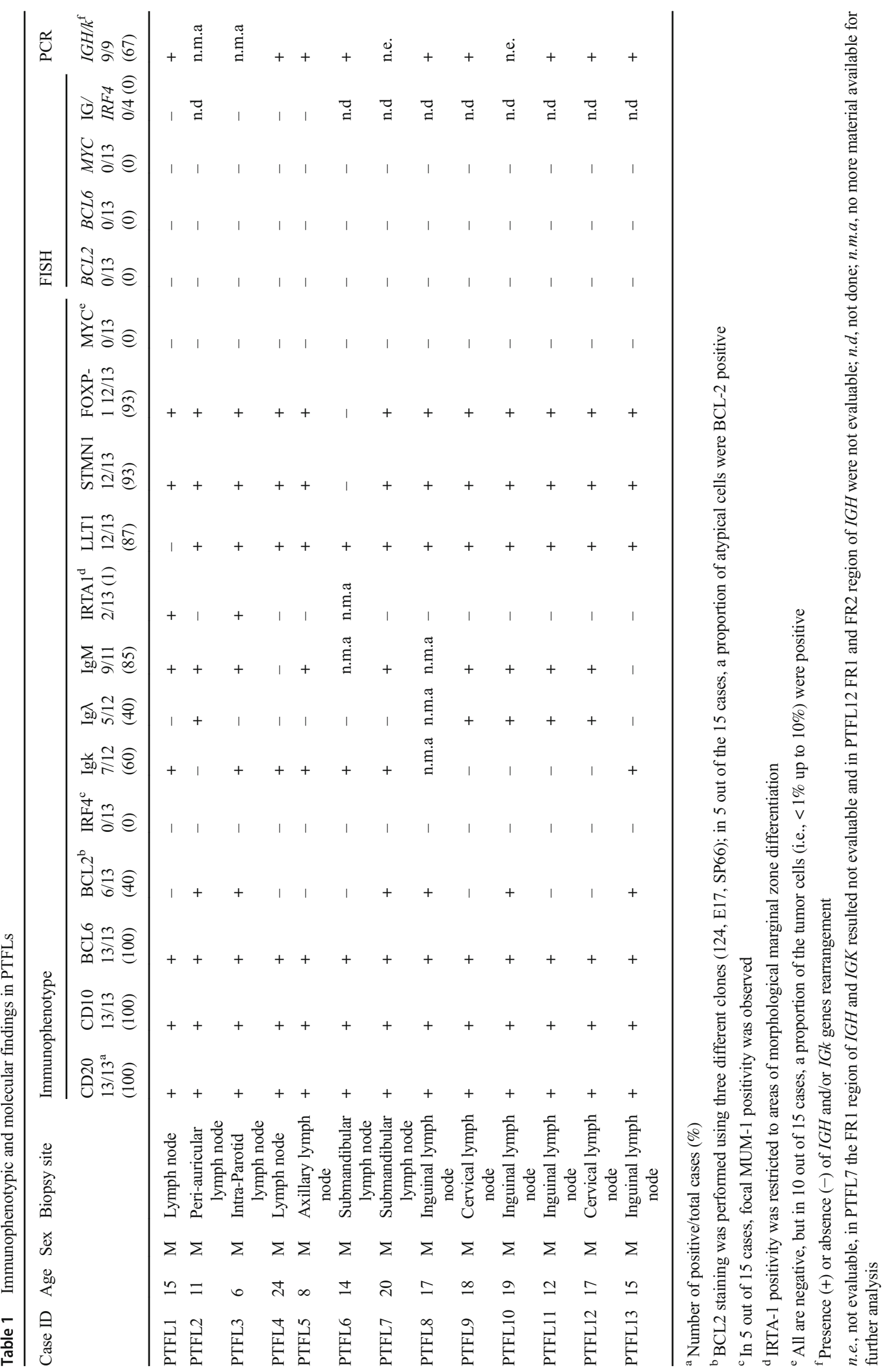


Fig. 1 a PTFL in a lymph node showing the characteristic expanded, irregular, and often closely packed lymphoid follicles with serpiginous outlines and attenuated mantle zones (hematoxylin and eosin; $\times 10$ ). The GCs contain a monotonous proliferation of medium- to largesized blastoid cells with round/ oval nuclei, finely clumped chromatin, and small nucleoli (inset; hematoxylin and eosin; $\times 40$ ). b The black arrows indicate small reactive follicles adjacent to the follicular proliferation. This lowpower image illustrates the socalled "a node within a node" appearance (hematoxylin and eo$\sin ; \times 2$ ). $\mathbf{c}$ The neoplastic follicles are strongly and homogeneously positive for CD20, CD10 (d), and BCL6 $(\mathbf{e})(\times 200)$. f The GCs in PTFL lack strong BCL2 protein expression $(\times 200)$. $g$ The enlarged follicles show attenuated IgD-positive mantle zones ( $\times$ 200). h, i Light chain staining of an abnormal germinal center in PTFL showing positive staining for kappa light chains (h) and negative lambda staining (i) (at both $\times 20$ and $\times 200$

magnifications)
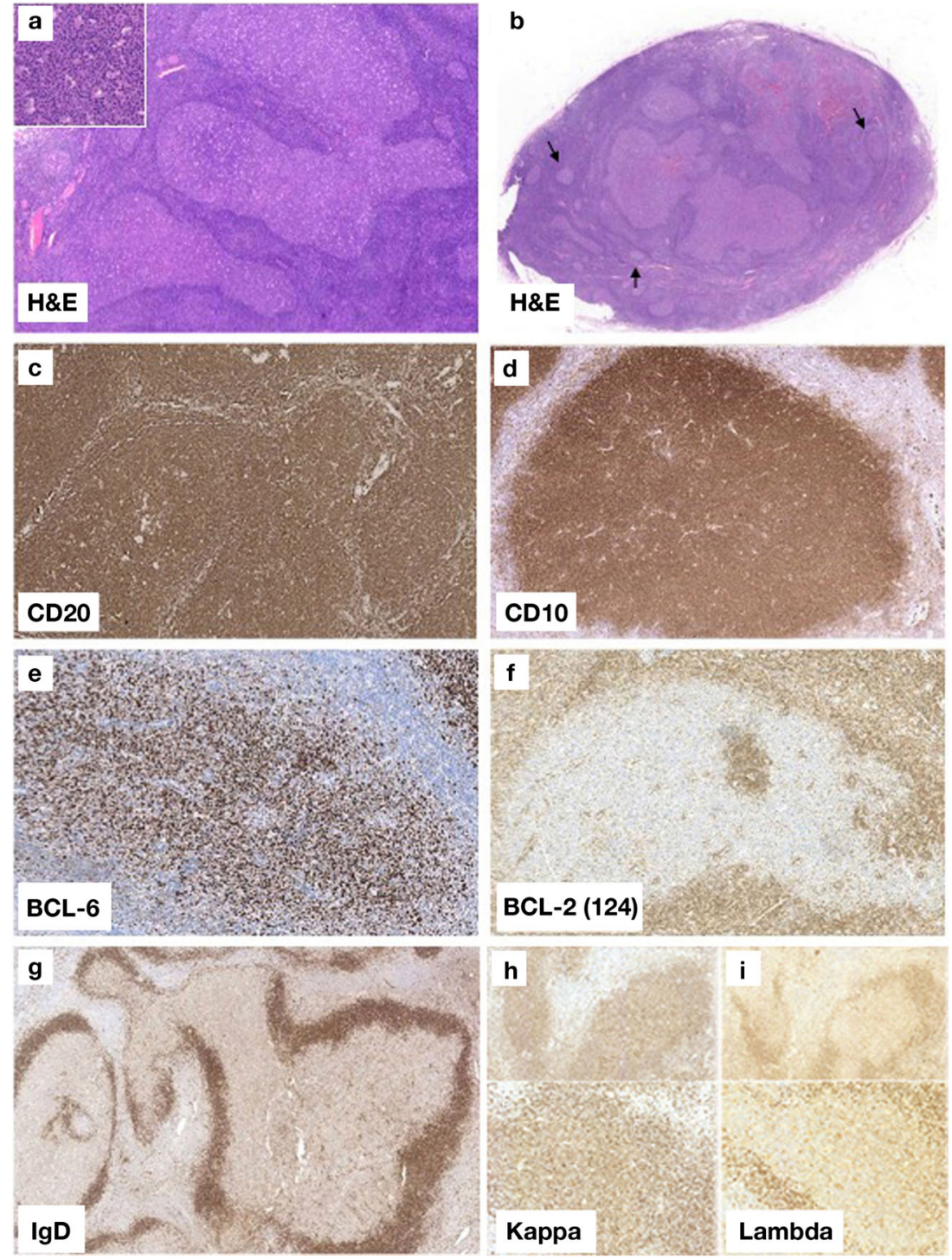

9/11 (81\%) cases, and kappa or lambda light-chain restriction in $7 / 12(60 \%)$ and 5/12 (40\%), respectively (Fig. 1h, i).

The germinal center-associated molecules LLT-1 and STMN-1 were both expressed in 12/13 (92\%) (Fig. 2a, b); the STMN-1-negative (PTFL6) and the LLT-1negative (PTFL1) cases both showed a monoclonal IG gene rearrangement. The marginal zone marker IRTA-1 was focally detected in $2 / 13(15 \%)$ of the cases in areas of "monocytoid differentiation" at the periphery of follicles. Strong nuclear staining for the transcription factor FOXP-1 in $>80 \%$ cells was found in $12 / 13(92 \%)$ of PTFLs (Fig. 2c). The FOXP-1-negative case (PTCL6) carried a clonal rearrangement of the IG genes.

In contrast, investigation of FOXP-1 expression in the 20 lymph nodes with $\mathrm{FH}$ showed that no cases exhibited FOXP-1 positivity in the reactive germinal centers (Fig. 2d).

\section{Genetics}

In eleven cases, the IG rearrangement status was investigated by PCR. In 9/9 patients, IGH and/or IGK gene rearrangements were detected. In PTFL7 and PTFL10, IG analysis yielded suboptimal amplification, with no defined clonal peaks (the FR1 region of IGH and IGK in PTFL7 and the FR1 and FR2 region of IGH in PTFL10 were not evaluable); however, PTFL 7 and PTFL10 showed $\mathrm{BCL}^{+} / \kappa^{+}$and $\mathrm{BCL} 2^{+} / \lambda^{+}$germinal centers respectively. In the remaining two samples (PTFL2 and PTFL3), no further material for molecular studies was available, but the GCs respectively stained as $\mathrm{BCL} 2^{+} / \lambda^{+}$ and $\mathrm{BCL}^{+} / \kappa^{+}$.

FISH performed in 13 cases disclosed no rearrangements of BCL2, BCL6, and MYC. Four cases were investigated also with IGH/IRF4 fusion probes, but no translocations were detected. 
Fig. 2 Neoplastic GCs in PTFL expressing a STMN-1 $(\times 100)$ and $\mathbf{b}$ LLT $-1(\times 100)$. c Strong nuclear staining for FOXP-1 in PTFL germinal centers. The black arrow highlights an included reactive $\mathrm{GC}$ which is negative with this marker $(\times 100)$. d Lymph node from a case of FH. The expanded germinal centers are negative for FOXP-1 $(\times 100)$
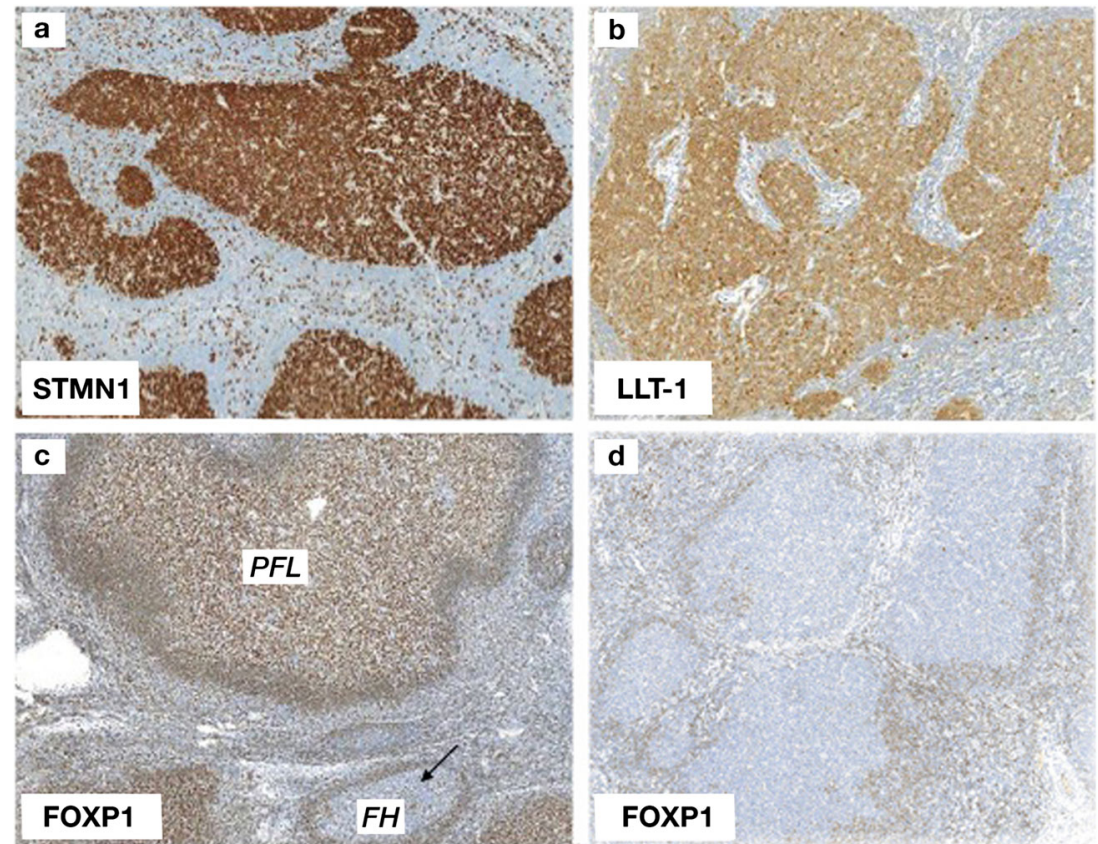

\section{Discussion}

PTFL is an uncommon clinicopathological entity newly recognized in the revised 4th edition of the WHO Classification of Tumours of Haematopoietic and Lymphoid Tissues. It predominantly occurs in young patients and only rarely in the elderly and differs from conventional FL on morphologic, genetic, and clinical grounds [1]. In line with previous reports [2-11], in our series, age at onset ranged between 6 and 24 years with a median age of 15 years. Our study also confirmed the strong association with male gender and the unique histological and phenotypic features of PTFL [2-11].

Recent studies have successfully delineated the molecular profile of PTFL $[10-12,16]$. Two independent groups performed a genome-wide analysis demonstrating that when compared with conventional $\mathrm{t}(14 ; 18)^{+}$and $\mathrm{t}(14 ; 18)^{-} \mathrm{FL}$, nodal PTFL is characterized by low genomic complexity (which fits well with the extremely indolent clinical behavior) and rare or absence of the characteristic mutations in the epigenetic modifiers CREBBP, EZH2, and KMT2D [10-12]. Recurrent mutations/deletions of TNFRSF 14 and mutations of MAP2K1 genes were found in 54\% and $49 \%$ of the samples respectively; these were mutually exclusive in the majority of the cases [10-12]. Furthermore, mutation of the IRF8 gene was reported in a subset of patients $(15 \%){ }^{12,16}$. These most frequent genetic aberrations might play a pivotal role in the pathogenesis of the disease, altering pathways associated with immune escape, apoptosis, and the germinal center reaction [10-12].

In a diagnostic setting, the main histological differential diagnoses are florid follicular hyperplasia (FFH) (given the expanded GCs and lack of strong BCL2 expression), pediatric marginal zone lymphoma (PMZL) which shows morphological and clinical similarities, $\mathrm{t}(14 ; 18)^{-} \mathrm{FL}$ where the age at presentation may overlap, and LBCL with IRF4 rearrangements. The latter condition occurs in the same age group as PTFL; it often involves Waldeyer's ring and can be also be seen in head and neck lymph nodes and the gastrointestinal tract, and may follow a more aggressive clinical course [13-15]. One morphological feature that is helpful in differentiating PTFL from FFH is the identification of a monotonous population of blastoid cells in the expanded GCs in PTFL; FFH shows polarized follicles composed of centroblasts and centrocytes [1]. Additionally, the germinal center cells in PTFL show very strong CD10 and BCL6 expression; staining is usually stronger than that seen in the residual GCs at the periphery of the node. These residual GCs contrast with the large irregular GC in PTFL, giving the characteristic "node within a node" appearance. Clonality analysis is mandatory for the diagnosis of PTFL. Accordingly, when evaluable, all our cases showed IGH and/or IGk rearrangement and monotypic restriction for immunoglobulin light chains.

Given the above-mentioned challenges in the diagnosis of PTFL, current study included an assessment of novel biomarkers for potential application in this diagnostic setting. Based on our data, a phenotypic feature that consistently differentiated PTFL from FFH was the expression of the FOX-P1 protein, which we found in most PTFL cases of our series, but not in the cases of FFH. The FOXP-1 (Forkhead Box P1) gene located at $3 \mathrm{p} 14.1$ codes for a homologous transcriptional regulator (FOXP-1), belonging to the FOX transcription factor family, which is implicated in a wide range of biological processes, including B cell development and immune response regulation. FOXP-1 is downregulated in germinal centers, its expression 
being inversely related to BCL6 expression and also has been reported to repress plasma cell differentiation [22, 23]. It cooperates with NF-kB signaling to promote expansion of primary mature human B cells [24]. In transgenic mice, constitutive FOXP-1 expression impairs GC formation and function, which might contribute to B cell lymphomagenesis [22]. FOXP-1 positivity has been reported in FL, MZL, and primary testicular lymphomas and represents a prognostic marker in a subset of large B cell lymphomas [25-34]. FOXP-1 overexpression has been reported in association with $\mathrm{t}(3 ; 14)(\mathrm{p} 14.1 ; \mathrm{q} 32)$, leading to a FOXP-1-IGH fusion and in rearrangements with non-IG partners, causing the expression of the $\mathrm{N}$-truncated isoforms, or in trisomy 3 [25-34]. Recently, Mottok et al. [35] demonstrated that immunohistochemical expression of FOXP-1 predicted adverse failure-free survival in conventional FL treated with immunochemotherapy. They found FOXP-1 to be significantly downregulated in both $E Z H 2$ - and $M E F 2 B$-mutated cases and that high FOXP-1 expression was associated with distinct molecular features such as TP53 mutations, expression of IRF4, and gene expression signatures reminiscent of dark zone germinal center or activated B cells [35].

We demonstrated strong nuclear staining in $>80 \%$ of cells for the transcription factor FOXP-1 in 93\% of our PTFLs while none of the FH cases analyzed showed FOXP-1 positivity in the reactive GCs. The FOXP-1 expression in PTFL was also confirmed at the RNA level by RNAscope in 5 cases (unpublished data). The level of FOXP-1 positivity in PTFL was higher than the one observed by Mottok et al. [35] in conventional FL: they reported $40 \%$ of FOXP- $1^{+}$cases, applying a cutoff positivity of $10 \%$. This discrepancy could be related to differences in sensitivity in the detection systems used in the two studies or might reflect the biological differences between PTFL and FL in adults as previously delineated by Schmidt at al. [10].

Moreover, we identified in PTFL aberrant co-expression of FOXP-1 and BCL6 protein, proteins that are normally reciprocally expressed in the GC [22]. The presence of the genetic aberration(s) sustaining FOXP-1 overexpression in PTFL needs to be investigated in further studies. However, our data supports the use of FOXP-1 as a candidate marker of neoplastic transformation and an essential tool for differentiating PTFL from FH.

The differential diagnosis between PTFL and PMZL can be challenging due to the shared features (male predominance, localized disease, excellent prognosis) [36]. On morphologic grounds, progressively transformed germinal center-like changes and residual hyperplastic GCs maintaining a starrysky appearance as well as polarization suggest the diagnosis of PMZL $[4,6,36]$. PMZL is also characterized by expanded interfollicular areas consisting of $\mathrm{CD} 20^{+} \mathrm{B}$ cells and the frequent negative immunohistochemical staining for CD10 and BCL6 [4, 6, 36]. The lack of interfollicular involvement by atypical $\mathrm{CD} 20^{+}$cells in our cases, which is highly characteristic of PTFL, facilitated the distinction from PMZL. Furthermore, most nodal and extranodal marginal zone lymphomas express IRTA- $1^{+}$[37], a protein only focally detected in 2/13 (15\%) of our PTFLs where there were features indicating a degree of marginal zone differentiation. The diagnosis of PTFL was also favored by the expression of the GC-associated molecules STMN1 and LLT-1 in 12/13 (92\%) and in $12 / 13(92 \%)$ of the cases respectively. STMN1 is an intracellular protein involved in multiple cell signaling pathways and is a GC-associated marker [38]. Previous studies of STMN1 expression in solid and hematological malignancies have shown an association with tumor behavior and prognosis [39-42]. In addition, we previously demonstrated the diagnostic relevance of STMN1 for FLs including those that are BCL2 negative, and showed its potential usefulness in distinguishing FLs from marginal zone lymphomas (MZLs), which are usually STMN1 negative [38].

LLT-1 is known to be present in GC-associated B cells and early plasmablasts. Its expression is readily induced via BCR, CD40, and CpG stimulation on B cells. The LLT1 ligand, CD161, was found to be highly expressed on follicular dendritic cells suggesting that LLT1-CD161 interactions play a novel and important role in B cell maturation [43]. LLT1 expression was seen on GC-derived lymphomas including Burkitt lymphoma (73\%), conventional FL (51\%), and lymphocyte predominant Hodgkin lymphoma (44\%) ${ }^{43}$. Our data suggest that STMN1 and LLT1 are maintained during malignant transformation in cases of PTFL and may be useful additional diagnostic markers in the differential diagnosis between PTFL and PMZL.

In summary, in this study, we reported a series of PTFL cases and demonstrated that the expression of novel GCassociated markers LLT-1 and STMN-1 is helpful in the differential diagnosis with PMZL. Additionally, we demonstrated that in contrast to FH, FOXP-1 is consistently expressed in the germinal centers in PTFL. Since FOXP-1 is not expressed in reactive $\mathrm{GC}$, it emerges as a new diagnostic marker for PTFL and is particularly helpful in differentiating this condition from florid $\mathrm{FH}$.

Author's contributions TM, ES, SAP, and LQM conceived and designed the study; CA, TM, AA, ES, AM, HR, MRJ, SP, IP, DL, SD, and TM wrote, edited, and reviewed the manuscript. AA, TM, ES, LQM, and SP researched and analyzed data. All authors gave final approval for publication. TM takes full responsibility for the work as a whole, including the study design, access to data, and the decision to submit and publish the manuscript.

Funding This manuscript was in part financially supported by the AIRC $5 \times 1000$ grant no. 21198 to SAP.

\section{Compliance with ethical standards}

The present study was conducted according to the principles of the Helsinki declaration after approval of the internal review board, the 
National Research Ethics Service, Research Ethics Committee 4 (REC Reference number 09/H0715/64).

Conflict of interest The authors declare that they have no conflict of interest.

Open Access This article is distributed under the terms of the Creative Commons Attribution 4.0 International License (http:// creativecommons.org/licenses/by/4.0/), which permits unrestricted use, distribution, and reproduction in any medium, provided you give appropriate credit to the original author(s) and the source, provide a link to the Creative Commons license, and indicate if changes were made.

\section{References}

1. Jaffe ES, Harris NL, Siebert R et al (2017) Paediatric-type follicular lymphoma. In: Swerdlow SH, Campo E, Harris NL et al (eds) WHO Classification of Tumours of Haematopoietic and Lymphoid Tissues. IARC Press, Lyon, pp 278-279

2. Atra A, Meller ST, Stevens RS et al (1998) Conservative management of follicular non-Hodgkin's lymphoma in childhood. Br J Haematol 103:220-223

3. Lorsbach RB, Shay-Seymore D, Moore J et al (2002) Clinicopathologic analysis of follicular lymphoma occurring in children. Blood 99:1959-1964

4. Swerdlow SH (2004) Paediatric type follicular lymphomas, marginal zone lymphomas, and marginal zone hyperplasia. Am J Clin Pathol (122 Suppl):S98-S109

5. Louissaint A Jr, Ackerman AM, Dias-Santagata D et al (2012) Paediatric-type nodal follicular lymphoma: an indolent clonal proliferation in children and adults with high proliferation index and no BCL2 rearrangement. Blood 120:2395-2404

6. Liu Q, Salaverria I, Pittaluga S et al (2013) Follicular lymphomas in children and young adults: a comparison of the paediatric type variant with usual follicular lymphoma. Am J Surg Pathol 37: 333-343

7. Oschlies I, Salaverria I, Mahn F et al (2010) Paediatric type follicular lymphoma-a clinico-pathological study of a population-based series of patients treated within the non-Hodgkin's lymphomaBerlin-Frankfurt-Munster (NHL-BFM) multicenter trials. Haematologica 95:253-259

8. Quintanilla-Martinez L, Sander B, Chan JK et al (2016) Indolent lymphomas in the paediatric type population: follicular lymphoma, IRF4/MUM1+ lymphoma, nodal marginal zone lymphoma and chronic lymphocytic leukemia. Virchows Arch 468:141-157

9. Attarbaschi A, Beishuizen A, Mann G, et al Children and adolescents with follicular lymphoma have an excellent prognosis with either limited chemotherapy or with a "watch and wait" strategy after complete resection. Ann Hematol 2013; 92: 1537-1541

10. Schmidt J, Gong S, Marafioti T et al (2016) Genome-wide analysis of pediatric-type follicular lymphoma reveals low genetic complexity and recurrent alterations of TNFRSF14 gene. Blood. 128:11011111

11. Louissaint A Jr, Schafernak KT, Geyer JT et al (2016) Pediatric-type nodal follicular lymphoma: a biologically distinct lymphoma with frequent MAPK pathway mutations. Blood. 128:1093-1100

12. Schmidt J, Ramis-Zaldivar JE, Nadeu F, et al Mutations of $M A P 2 K 1$ are frequent in pediatric-type follicular lymphoma and result in ERK pathway activation. Blood. 2017; 130: 323-327

13. Pittaluga S, Harris NL, Siebert R et al (2017) Large B-cell lymphoma with IRF4 rearrangement. In: Swerdlow SH, Campo E, Harris
NL et al (eds) WHO Classification of Tumours of Haematopoietic and Lymphoid Tissues. IARC Press, Lyon, pp 280-281

14. Pittaluga S, Harris NL, Siebert R et al (2017) IRF4 B-cell lymphoma with IRF4 rearrangment. In: Swerdlow SH, Campo E, Harris NL et al (eds) WHO Classification of Tumours of Haematopoietic and Lymphoid Tissues. IARC Press, Lyon, pp 280-281

15. Salaverria I, Philipp C, Oschlies I et al (2011) Translocations activating IRF4 identify a subtype of germinal center-derived B-cell lymphoma affecting predominantly children and young adults. Blood 118:139-147

16. Ozawa MG, Bhaduri A, Chisholm KM et al A study of the mutational landscape of pediatric-type follicular lymphoma and pediatric nodal marginal zone lymphoma. Mod Pathol 2016; 29(10):12121220

17. Marafioti T, Paterson JC, Ballabio E et al (2008) Novel markers of normal and neoplastic human plasmacytoid dendritic cells. Blood 111:3778-3792

18. Hans CP, Weisenburger DD, Greiner TC, et al Confirmation of the molecular classification of diffuse large B-cell lymphoma by immunohistochemistry using a tissue microarray. Blood 2004 Jan 1;103(1):275-282

19. Haralambieva E, Banham AH, Bastard C et al (2003) Detection by the fluorescence in situ hybridization technique of MYC translocations in paraffin-embedded lymphoma biopsy samples. Br J Haematol 121:49-56

20. Ventura RA, Martin-Subero JI, Jones M et al (2006) FISH analysis for the detection of lymphoma-associated chromosomal abnormalities in routine paraffin-embedded tissue. J Mol Diagn 8:141-151

21. Gazzola A, Mannu C, Rossi M et al (2014) The evolution of clonality testing in the diagnosis and monitoring of hematological malignancies. Ther Adv Hematol 5:35-47

22. Sagardoy A, Martinez-Ferrandis JI, Roa S et al (2013) Downregulation of FOXP-1 is required during germinal center Bcell function. Blood. 121:4311-4320

23. van Keimpema M, Grüneberg LJ, Mokry M et al (2015) The forkhead transcription factor FOXP-1 represses human plasma cell differentiation. Blood. 126:2098-2109

24. van Keimpema M, Grüneberg LJ, Mokry M et al (2014) FOXP-1 directly represses transcription of proapoptotic genes and cooperates with NF-KB to promote ssurvival of human B cells. Blood. 124:3431-3440

25. Brown P, Marafioti T, Kusec R et al (2005) The FOXP-1 transcription factor is expressed in the majority of follicular lymphomas but is rarely expressed in classical and lymphocyte predominant Hodgkin's lymphoma. J Mol Histol 36:249-256

26. Barrans SL, Fenton JA, Banham A, Owen RG, Jack AS (2004) Strong expression of FOXP-1 identifies a distinct subset of diffuse large B-cell lymphoma (DLBCL) patients with poor outcome. Blood. 104:2933-2935

27. Banham AH, Connors JM, Brown PJ et al (2005) Expression of the FOXP-1 transcription factor is strongly associated with inferior survival in patients with diffuse large B-cell lymphoma. Clin Cancer Res 11:1065-1072

28. Goatly A, Bacon CM, Nakamura S et al (2008) FOXP-1 abnormalities in lymphoma: translocation breakpoint mapping reveals insights into deregulated transcriptional control. Mod Pathol 21: 902-911

29. Streubel B, Vinatzer U, Lamprecht A et al (2005) $\mathrm{T}(3 ; 14)(\mathrm{p} 14.1 ; \mathrm{q} 32)$ involving IGH and FOXP-1 is a novel recurrent chromosomal aberration in MALT lymphoma. Leukemia. 19: 652-658

30. Wlodarska I, Veyt E, de Paepe P et al (2005) FOXP-1, a gene highly expressed in a subset of diffuse large B-cell lymphoma, is recurrently targeted by genomic aberrations. Leukemia 19:1299-1305

31. Haralambieva E, Adam P, Ventura R et al (2006) Genetic rearrangement of FOXP-1 is predominantly detected in a subset of diffuse 
large B-cell lymphomas with extranodal presentation. Leukemia 20:1300-1303

32. Courts C, Brunn A, Montesinos-Rongen M et al (2009) Preferential expression of truncated isoforms of FOXP-1 in primary central nervous system lymphoma. J Neuropathol Exp Neurol 68:972-976

33. Rouhigharabaei L, Finalet Ferreiro J, Tousseyn T et al (2014) NonIG aberrations of FOXP-1 in B-cell malignancies lead to an aberrant expression of N-truncated isoforms of FOXP-1. PLoS One 9:85851

34. Twa DD, Mottok A, Chan FC et al (2015) Recurrent genomic rearrangements in primary testicular lymphoma. J Pathol 236: 136-141

35. Mottok A, Jurinovic V, Farinha P et al (2018) FOXP-1 expression is a prognostic biomarker in follicular lymphoma treated with rituximab and chemotherapy. Blood 131:226-235

36. Taddesse-Heath L, Pittaluga S, Sorbara L et al (2003) Marginal zone B-cell lymphoma in children and young adults. Am J Surg Pathol 27:522-531

37. Falini B, Agostinelli C, Bigerna B et al (2012) IRTA1 is selectively expressed in nodal and extranodal marginal zone lymphomas. Histopathology 61:930-941

38. Marafioti T, Copie-Bergman C, Calaminici M et al (2013) Another look at follicular lymphoma: immunophenotypic and molecular analyses identify distinct follicular lymphoma subgroups. Histopathology 62:860-875

39. Hanash SM, Strahler JR, Kuick R et al (1988) Identification of a polypeptide associated with the malignant phenotype in acute leukemia. J Biol Chem 263:12813-12815

40. Melhem RF, Zhu XX, Hailat N et al (1991) Characterization of the gene for a proliferation-related phosphoprotein (oncoprotein 18) expressed in high amounts in acute leukemia. J Biol Chem 266: 17747-17753

41. Gez S, Crossett B, Christopherson RI (2007) Differentially expressed cytosolic proteins in human leukemia and lymphoma cell lines correlate with lineages and functions. Biochim Biophys Acta 1774:1173-1183

42. Belletti B, Baldassarre G (2011) Stathmin: a protein with many tasks. New biomarker and potential target in cancer. Expert Opin. Ther Targets 15:1249-1266

43. Llibre A, López-Macías C, Marafioti T et al (2016) LLT1 and CD161 expression in human germinal centers promotes B cell activation and CXCR4 downregulation. J Immunol 1(96):2085-2094

Publisher's note Springer Nature remains neutral with regard to jurisdictional claims in published maps and institutional affiliations. 